\title{
DIABETES
}

\section{Attenuated incretin effect after glucose loading in monogenic HNF1A diabetes}

Maturity-onset diabetes of the young (MODY), also known as monogenic diabetes mellitus, is a group of hereditary forms of nonautoimmune diabetes mellitus characterized by late-onset in early adulthood. MODY2 and MODY3caused by mutations in the genes encoding glucokinase (GCK) and hepatocyte nuclear factor $1 \alpha(\mathrm{HNF} 1 \mathrm{~A})$, respectively-are the two most common forms. In a new study, Signe Østoft and colleagues report that patients with MODY3 exhibit a diminished incretin effect.

The incretin effect-defined as augmentation of the insulin response by gastrointestinal hormones after oral glucose intake compared with administration of glucose intravenously-is impaired in type 2 diabetes mellitus, but has been little studied in monogenic diabetes mellitus. The researchers used isoglycaemic intravenous glucose infusion (IGII) to evaluate the incretin effect in patients with MODY2 and MODY3, compared with healthy individuals with no family history of diabetes mellitus.

Fasting plasma glucose levels were higher in patients with MODY2 and MODY3 than in control individuals, with glucose intolerance being most pronounced in those with MODY3. Patients with MODY3 exhibited significantly lower insulin and C-peptide responses during both OGTT and IGII than patients with MODY2 or control individuals. The incretin effect-calculated from either insulin release or C-peptide levels-was attenuated in patients with MODY3 but not in patients with MODY2. Normal insulin resistance, as defined by HOMA, was found in all groups. Following OGTT, control individuals and patients with MODY2 exhibited normal suppression of glucagon secretion. By contrast, glucagon secretion initially increased in patients with MODY3, subsequently decreasing to levels lower that those observed after IGII. Incretin

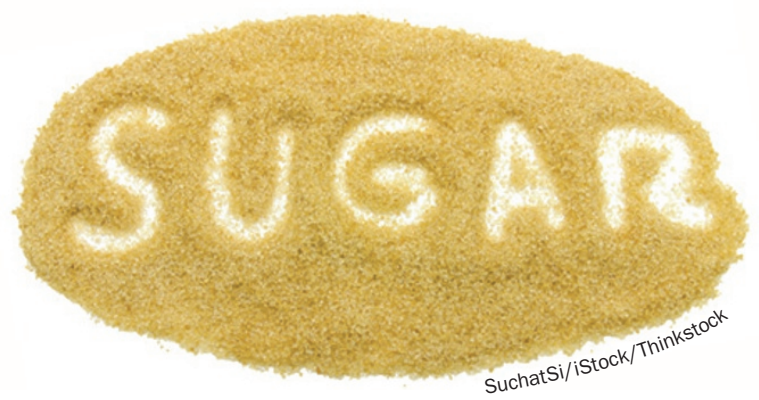

hormone responses were normal in all study participants.

"Patients with MODY2 exhibit preserved incretin physiology, whereas patients with MODY3 exhibit a reduced incretin effect, $\beta$-cell dysfunction and an inappropriate glucagon response to OGTT and, as such, resemble patients with type 2 diabetes mellitus," summarizes Østoft. Randomized controlled trials are warranted to assess whether incretinbased therapies might be useful in treating patients with MODY3.

\section{David Holmes}

Original article Østoft, S. H. et al. Incretin effect and glucagon responses to oral and intravenous glucose in patients with maturity onset diabetes of the young-type 2 and type 3. Diabetes doi:10.2337/db13-1878 